\title{
Free energy of cylindrical polyions: analytical results
}

\author{
Gabriel Téllez \\ Departamento de Fúsica, Universidad de los Andes, Bogotá, Colombi米 \\ Emmanuel Trizac \\ Laboratoire de Physique Théorique et Modèles Statistiques (UMR CNRS 8626), \\ Université Paris-Sud, Université Paris Saclay, F-91405 Orsay, Franc@
}

\begin{abstract}
Within the Poisson-Boltzmann (PB) framework useful for a wealth of charged soft matter problems, we work out the Coulombic grand potential of a long cylindrical charged polyion in a binary electrolyte solution of arbitrary valency and for low salt concentration. We obtain the exact analytical low-salt asymptotic expression for the grand potential, derived from known properties of the exact solutions to the cylindrical PB equation. These results are relevant for understanding nucleic acid processes. In practice, our expressions are accurate for arbitrary polyion charges, provided their radius is smaller than the Debye length defined by the electrolyte.
\end{abstract}

\section{INTRODUCTION}

Coulombic interactions play an important role in the physico-chemical and thermodynamic properties of highly charged polyions in a solution with added salt [1-3]. In this work, we concentrate on free energy calculations pertaining to stiff charged polymers, essential for a number of applications among which melting and binding of biopolymers such as nucleic acids. We provide analytical expressions for the free energy of the formation of the electrical double layer around long cylindrical polyions such as deoxyribonucleic acid (DNA), in the infinite dilution limit. On the mean field level, where correlations are neglected, the nonlinear Poisson-Boltzmann (PB) theory provides accurate predictions for most applications [4 -7], which can be tested against results from Monte-Carlo and molecular dynamics simulations [8, 9]. Nowadays, with available computer numerical libraries and programs, it is fairly straightforward to solve numerically PB equation to obtain many quantities of interest, including the free energy, for example see [10] and appendix $\mathrm{A}$ below. However, it is desirable to obtain analytic results that give more insight into the dependency of the free energy on the parameters of the system: the linear charge of the polyion, the salt concentration and ions valencies. For high salt concentration situations, an expansion in small curvature around the planar double layer result can be build to provide results for the free energy [11]. Here, we will concentrate on the opposite regime of low salt concentration, making use of the known analytic asymptotic expansion of the solution of PB equation 12 17. Due to the chemical equilibrium with the reservoir, the appropriate ensemble is the grand-canonical one. Therefore we will concentrate on evaluating the grand potential. The free energy can be obtained by the usual Legendre transformation, see appendix B.

This work is organized as follows. In Sec. II after recalling the PB framework and previous results, we derive the exact low-salt concentration asymptotic analytic expression for the grand potential. The result is valid for any value of the linear charge of the polyion. In Sec. III, we provide a simplification of the general result that is valid for moderate to highly charged polyions. This expression has the advantage to be valid for any electrolyte valencies and not limited to 1:1. Finally in Sec. IV] we benchmark our analytic expressions against numerical evaluation of the grand potential and we discuss our predictions dependence on the polyion charge, on the salt concentration and on the electrolyte valencies.

\section{GENERAL EXPRESSION FOR THE GRAND POTENTIAL}

Our framework is the nonlinear PB equation [1 [3] to describe a cylindrical polyion in an infinite electrolyte medium with dielectric permittivity $\epsilon$. The persistence length of the polyion is supposed to be much larger than all other physical lengths of interest, therefore the polyion is modeled as an infinite cylinder of radius $a$ with uniform linear charge density $\lambda=-e / b<0$, with $e>0$ the elementary charge and $b$ the longitudinal distance per unit charge. The system is in thermal and chemical equilibrium with a salt reservoir at temperature $T=1 /\left(k_{B} \beta\right)\left(k_{B}\right.$ is Boltzmann

\footnotetext{
* gtellez@uniandes.edu.co (corresponding author)

$\dagger$ trizac@lptms.u-psud.fr
} 
constant) and chemical potentials $\mu_{ \pm}=k_{B} T \ln \left(n_{ \pm}^{0} \Lambda_{ \pm}^{3}\right)$, where $n_{ \pm}^{0}$ are the ionic bulk densities and $\Lambda_{ \pm}$are the de Broglie thermal wavelengths of the ions. The electrolyte valencies are $z_{-}: z_{+}$. Both numbers are taken positive with the convention of writing first the coion valency (here $\left.z_{-}\right)$then the counterion valency $\left(z_{+}\right)$. The charge density of the polyion can be characterized by the dimensionless parameter $\xi=l_{B} / b=-\lambda l_{B} / e>0$, with $l_{B}=\beta e^{2} / \epsilon$ the Bjerrum length (around $0.71 \mathrm{~nm}$ for water at room temperature). The solvent (water) is modeled as a continuous medium of dielectric relative permitivitty $\epsilon$. The Debye length $\kappa^{-1}$ is defined by $\kappa^{2}=4 \pi l_{B}\left(z_{+}^{2} n_{+}^{0}+z_{-}^{2} n_{-}^{0}\right)$. The dimensionless electrostatic potential at a radial distance $r$ from the polyion, $\phi(r)=\beta e y(r)$ (with $y(r)$ the electrostatic potential), satisfies $\mathrm{PB}$ equation

$$
\frac{1}{r} \frac{d}{d r}\left(r \frac{d \phi}{d r}\right)=\frac{\kappa^{2}}{z_{+}+z_{-}}\left(e^{z_{-} \phi(r)}-e^{-z_{+} \phi(r)}\right),
$$

with boundary conditions $a \phi^{\prime}(a)=2 \xi$ (Gauss law at contact with the polyion) and $\lim _{r \rightarrow \infty} r \phi^{\prime}(r)=0$ (electroneutrality of the system in the infinite dilution limit considered here). The grand potential of the system can be obtained by using one of several charging processes as recalled in appendix B [18 20]. We use here a charging process where $\xi$ is varied from 0 to its final value. The dimensionless excess grand potential per unit charge is $g=\omega / \xi$, with the dimensionless linear density of grand potential (see definitions (B4) and (B5) in appendix B)

$$
\omega=-\int_{0}^{\xi} \phi_{0} d \xi^{\prime}
$$

Here $\phi_{0}$ is the contact electrostatic potential $\phi_{0}=\phi(a)$ seen as a function of the charge $\xi^{\prime}$.

In this work we consider the low salt density regime when $\kappa a \ll 1$. As explained in appendix D of ref. [17], the short distance behavior of the electric potential can be obtained by injecting into PB Eq. (1) the o(1) approximation $\phi(r)=-2 A \ln (\kappa r)+\ln B+o(1)$ to compute higher order terms of powers $r . A$ and $B$ are constants of integration. Summing up all terms of order $r^{2 n\left(1+z_{+} A\right)}(n \in \mathbb{N})$ leads to the asymptotic expression when $\kappa a \ll 1$ for the contact potential (see Eq. (D6) from [17])

$$
\phi_{0}=z_{+}^{-1} \ln \left[\frac{(\kappa a)^{2} z_{+}}{2\left(z_{+}+z_{-}\right)}\left(\frac{\sin (\widetilde{\mu} \ln (\kappa a)+\Psi(\widetilde{\mu}))}{\widetilde{\mu}}\right)^{2}\right],
$$

where $\widetilde{\mu}$ is defined by $i \widetilde{\mu}=1+z_{+} A$ and

$$
\Psi(\widetilde{\mu})=-\frac{1}{2 i} \ln \frac{z_{+} B^{-z_{+}}}{8\left(z_{+}+z_{-}\right)(i \widetilde{\mu})^{2}} .
$$

This function has the property that $\Psi(0)=0$. To satisfy the boundary condition $\lim _{r \rightarrow \infty} r \phi^{\prime}(r)=0$ required by electroneutrality, the constant of integration $B$ is a function of $A$ and therefore of $\widetilde{\mu}$. The explicit form of $B$ and $\Psi(\widetilde{\mu})$ is only known in the cases of valencies $1: 1,1: 2$ and $2: 1$ where the connection problem of the long and short distances of the PB solution has been solved [12, 14] and those results are recalled in appendix C] However, for other valencies, we shall show that we only need to know the derivative at $0, \mathcal{C}=\Psi^{\prime}(0)$, and the definite integral $\mathcal{I}=\int_{-i}^{0} \Psi(u) d u$ to obtain information on the grand potential.

The other constant of integration, $A$, and therefore $\widetilde{\mu}$, are obtained by applying the boundary condition at the contact of the polyion $a \phi^{\prime}(a)=2 \xi$ which leads to

$$
z_{+} \xi-1=\widetilde{\mu} \cot (\widetilde{\mu} \ln (\kappa a)+\Psi(\widetilde{\mu}))
$$

For small charges $\xi \ll 1$ one has at order 0 in $\kappa a, A=-\xi$ and $\widetilde{\mu}=i\left(-1+z_{+} \xi\right)$. As $\xi$ increases, $\widetilde{\mu}$ moves on the imaginary axis from $-i$ to 0 where $\xi$ takes the critical value $\xi_{c}$ such that $\widetilde{\mu}=0$, given by

$$
z_{+} \xi_{c}-1=\frac{1}{\ln (\kappa a)+\mathcal{C}} .
$$

Notice that $\xi_{c}<1 / z_{+}$: as explained in [15], the effect of salt is to reduce the condensation threshold from $1 / z_{+}$to $\xi_{c}$. For $\xi>\xi_{c}$, the parameter $\mu$ becomes real and moves along the real axis up to the value [17]

$$
\widetilde{\mu}_{\infty}=\frac{-\pi}{\ln (\kappa a)+\mathcal{C}}
$$

as $\xi \rightarrow \infty$. The path followed by $\widetilde{\mu}$ is shown in Fig. 1, In past works [13, 15, 17], Eq. (3) was exclusively used in the region $\xi \geq \xi_{c}(\widetilde{\mu} \in \mathbb{R})$, however it should be clear from its derivation [13, 16, 17] that Eq. (33) is also valid when $\xi<\xi_{c}$ provided that $\widetilde{\mu}$ is imaginary. 


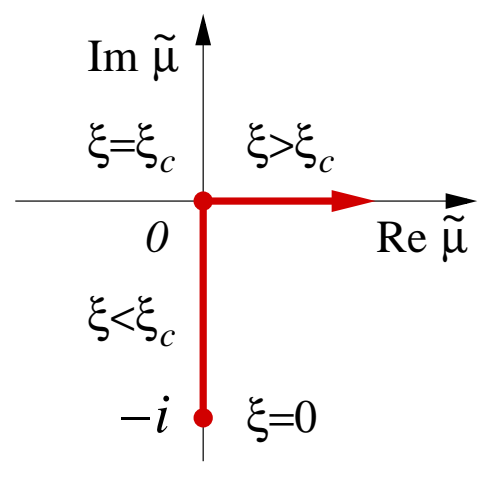

FIG. 1. Path followed by the parameter $\tilde{\mu}$ on the complex plane $\mathbb{C}$ as $\xi$ increases. For vanishing charge $(\xi=0), \widetilde{\mu}=-i$. Increasing $\xi, \widetilde{\mu}$ moves "up" along the imaginary axis, reaching $\widetilde{\mu}=0$ for $\xi=\xi_{c}$. For $\xi>\xi_{c}, \widetilde{\mu}$ is real and moves along the real axis; it is then a positive and non-decreasing function, see the arrow.

In this present work, we are interested in the situation with added salt. Nevertheless, it is interesting to comment on the similarities and differences of this case with the no-salt case, where there are only counterions in the solution. In Refs. 21, 22], the electrostatic potential in the no-salt case was worked out, which formally bears a close resemblance to Eq. (33) with $a$ replaced by $r$ and when the integration constants are identified: in Eq. (8) of Ref. [21], the integration constant $C$ plays the role of $\widetilde{\mu}$ and the integration constant $\ln A$ (from Ref. [21]) plays the role of $\Psi(\widetilde{\mu})$. The reason for this resemblance is that, at the close vicinity of the polyion, the coion density is negligible, thus the potential should behave asymptotically as the one of the no salt case. However, at large distances from the polyion, the expression for the potential becomes more involved in the case with added salt, and Eq. (3), with $a$ replaced by $r$, cannot be used at the edge of the Wigner-Seitz cell to determine the integration constant $\Psi(\widetilde{\mu})$ by applying the electroneutrality boundary condition, contrarily to the development done in the no-salt case [21]. Explicit expressions for this integration constant can only be obtained in the infinite dilution limit (infinite radius of the Wigner-Seitz cell) with the solution to the connection problem [12, 14].

Several approximations have been developed for solving Eq. (5D) [15 17] depending on the range of values of $\xi$ $\left(\xi<\xi_{c}\right.$ or $\left.\xi>\xi_{c}\right)$. Therefore it might prove difficult to perform the integral over $\xi$ in Eq. (2), as pointed out in [11]. Attempting such a calculation is indeed inconvenient. However, a change of variable from $\xi$ to $\widetilde{\mu}$ proves a useful reformulation. As we will show below, this leads to an indefinite integral that can be computed independently of the range of values considered for $\xi$.

First, we perform an integration by parts,

$$
\begin{aligned}
-\omega & =\int_{0}^{\xi} \phi_{0}\left(\xi^{\prime}\right) d \xi^{\prime}=\left(\xi-\frac{1}{z_{-}}\right) \phi_{0}-\int_{0}^{\phi_{0}}\left(\xi-\frac{1}{z_{+}}\right) d \phi_{0} \\
& =\left(\xi-\frac{1}{z_{-}}\right) \phi_{0}-\int_{-i}^{\widetilde{\mu}}\left(\xi-\frac{1}{z_{+}}\right) \frac{d \phi_{0}}{d \mu} d \mu,
\end{aligned}
$$

which can be interpreted as considering the thermodynamic potential appropriate for a fixed potential polyion rather than a fixed charge one [5]. In principle, the integral over $\widetilde{\mu}$ should follow the path shown in Fig. 1, however this is nonessential since the integrand is an holomorphic function of $\tilde{\mu}$ in the vicinity of the path considered in the complex plane. Now, from Eq. (3), we have

$$
\frac{d \phi_{0}}{d \widetilde{\mu}}=2 z_{+}^{-1}\left(\left(\ln (\kappa a)+\Psi^{\prime}(\widetilde{\mu})\right) \cot (\widetilde{\mu} \ln (\kappa a)+\Psi(\widetilde{\mu}))-\frac{1}{\widetilde{\mu}}\right)
$$

Using (5), it is useful to notice that

$$
\frac{d}{d \widetilde{\mu}}\left(z_{+} \xi-1\right)=\cot (\widetilde{\mu} \ln (\kappa a)+\Psi(\widetilde{\mu}))-\widetilde{\mu}\left(\ln (\kappa a)+\Psi^{\prime}(\widetilde{\mu})\right)\left(1+\cot ^{2}(\widetilde{\mu} \ln (\kappa a)+\Psi(\widetilde{\mu}))\right)
$$

With this, an exact differential appears in the integrand of (8)

$$
\left(\xi-\frac{1}{z_{+}}\right) \frac{d \phi_{0}}{d \widetilde{\mu}}=-2 z_{+}^{-1}\left[\frac{d}{d \widetilde{\mu}}\left(z_{+} \xi-1\right)+\widetilde{\mu}\left(\ln (\kappa a)+\Psi^{\prime}(\widetilde{\mu})\right)\right] .
$$


This yields

$$
\omega=-\left(\xi-\frac{1}{z_{+}}\right) \phi_{0}-\frac{2}{z_{+}} \xi-\frac{1}{z_{+}^{2}}\left[\left(\widetilde{\mu}^{2}+1\right) \ln (\kappa a)+2 \widetilde{\mu} \Psi(\widetilde{\mu})+2 i \Psi(-i)-2 \int_{-i}^{\widetilde{\mu}} \Psi(u) d u\right] .
$$

The value of $\Psi(-i)$ corresponds to the situation of an uncharged polyion with $B=1$ in (4). Then, $2 i \Psi(-i)=$ $-\ln \left(8 z_{+}^{-1}\left(z_{+}+z_{-}\right)\right)$. Because of relation (5), the following identity

$$
\left(z_{+} \xi-1\right)^{2}+\widetilde{\mu}^{2}=\left(\frac{\widetilde{\mu}}{\sin (\widetilde{\mu} \ln (\kappa a)+\Psi(\widetilde{\mu}))}\right)^{2}
$$

is satisfied. This can be used in (3) to obtain an alternative expression of the contact potential $\phi_{0}$, that when replaced into (12) gives

$$
\begin{aligned}
\omega & =-\frac{1}{z_{+}} \xi\left(2+\ln \frac{(\kappa a)^{2} z_{+}}{2\left(z_{+}+z_{-}\right)}\right)+\frac{1}{z_{+}}\left(\xi-\frac{1}{z_{+}}\right) \ln \left[\left(z_{+} \xi-1\right)^{2}+\widetilde{\mu}^{2}\right] \\
& +\frac{1}{z_{+}^{2}}\left(2 \ln 2+\left(1-\widetilde{\mu}^{2}\right) \ln (\kappa a)-2 \widetilde{\mu} \Psi(\widetilde{\mu})+2 \int_{-i}^{\widetilde{\mu}} \Psi(u) d u\right) .
\end{aligned}
$$

This is the general exact analytic asymptotic expression for the grand potential when $\kappa a \ll 1$ valid for all values of $\xi$. The parameter $\widetilde{\mu}$ is obtained by solving Eq. (5). In the following sections, we will develop some approximate solutions for Eq. (5) depending on the range of values of $\xi$ of interest.

It is worth noticing that the grand potential is an holomorphic function of $\widetilde{\mu}$ in the vicinity of the path shown in Fig. 1] in particular close and at $\widetilde{\mu}=0$ corresponding to $\xi=\xi_{c}$. Therefore, in the strict sense, there isn't any phase transition for any value of $\xi$ at any value of $\kappa a$. The grand potential changes smoothly with $\xi$, even in the region close to $\xi_{c}$ where the counterion condensation/de-condensation occurs. However, the change from imaginary $\widetilde{\mu}$ to real $\widetilde{\mu}$ does have quantitative implications on the small distance behavior of the electrostatic potential as it has been analyzed in [16]. For this reason, following tradition and with a slight abuse of language, we will refer to $\xi_{c}$ as the "critical" value for counterion condensation.

\section{RESULTS FOR MODERATE TO HIGHLY CHARGED POLYIONS}

In this section we develop a simplified expression of the previous result (14) that is valid for a wide range of charges $\xi$ which includes all the region $\xi \geq \xi_{c}$ but also part of the region below the critical value $\left(\xi<\xi_{c}\right)$ provided $\xi_{c}-\xi \ll 1$. This covers the most relevant range of values of $\xi$ for physico-chemical and biological applications, including the description of single (ss) and double stranded (ds) DNA where $\xi$ ranges between 2 and 4.2 .

For this range of values of $\xi$, the parameter $\tilde{\mu}$ is small. As in previous works, we perform a linearization of the function $\Psi(\widetilde{\mu}) \simeq \mathcal{C} \widetilde{\mu}$. Then Eq. (5) can be written as

$$
\frac{z_{+} \xi-1}{z_{+} \xi_{c}-1}=\widehat{\mu} \cot (\widehat{\mu})
$$

where we defined

$$
\widehat{\mu}=\widetilde{\mu}(\ln (\kappa a)+\mathcal{C})=\frac{\widetilde{\mu}}{z_{+} \xi_{c}-1} .
$$

Let $h$ be the inverse of the function $\widehat{\mu} \mapsto \widehat{\mu} \cot (\widehat{\mu})$ which can easily be tabulated numerically to any desired precision. Let

$$
\zeta=\frac{z_{+} \xi-1}{z_{+} \xi_{c}-1},
$$

then $\widehat{\mu}=h(\zeta)$, so that $\widetilde{\mu}$ is given by

$$
\widetilde{\mu}=\left(z_{+} \xi_{c}-1\right) h(\zeta)
$$

It should be noted that $h$ has several branches because Eq. (15) has an infinite number of solutions. We take the branch such that $h(1)=0$ (corresponding to $\widetilde{\mu}=0$ when $\xi=\xi_{c}$ ). Also, $h$ is to be considered as a complex function. 


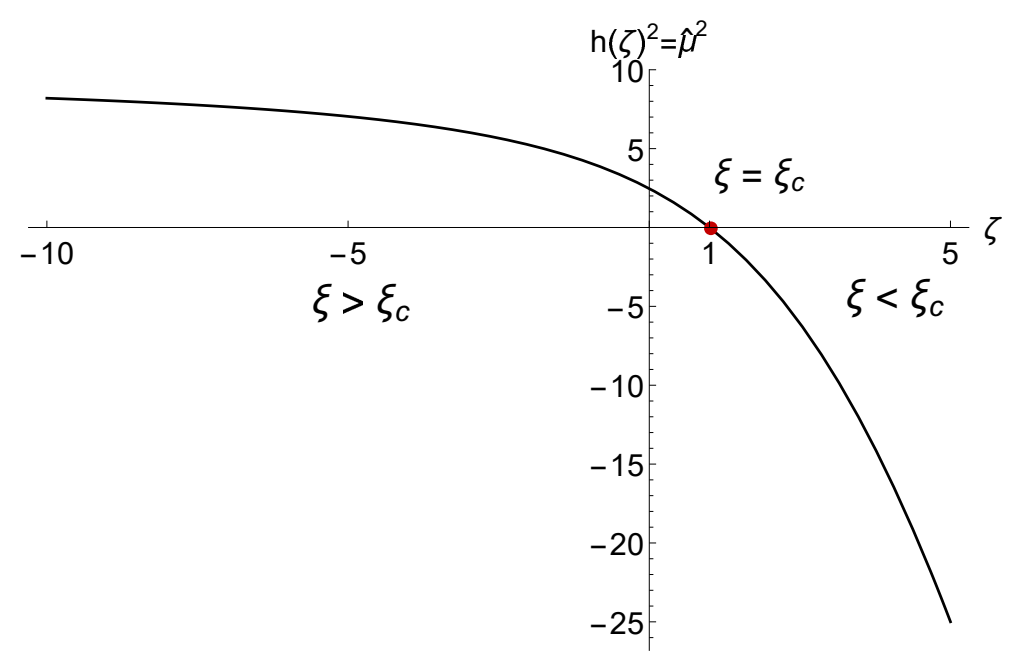

FIG. 2. The square of $h$, defined as the inverse function of $z \mapsto z \cot (z)$, which gives the value of $\widehat{\mu}^{2}$ as a function of $\zeta=\left(z_{+} \xi-1\right) /\left(z_{+} \xi_{c}-1\right)$.

When its argument $\zeta$ is real and $\zeta \leq 1, h(\zeta)$ is real, $\left(\widehat{\mu}^{2}=h(\zeta)^{2} \geq 0\right.$, corresponding to $\xi \geq \xi_{c}$ and $\left.\widetilde{\mu} \in\left[0, \widetilde{\mu}_{\infty}\right]\right)$. When $\zeta>1, h(\zeta)$ takes imaginary values $\left(\widehat{\mu}^{2}=h(\zeta)^{2}<0\right.$, corresponding to $\xi<\xi_{c}$ and $\left.\widetilde{\mu} \in[-i, 0]\right)$. Fig. 2 shows a plot of the square of the function $h$ which gives the value of $\widehat{\mu}^{2}$ as a function of $\zeta$.

The linearization of the function $\Psi$ around $\widetilde{\mu}=0$ leads to the following approximation for the grand potential (14)

$$
\begin{aligned}
\omega & =-\frac{1}{z_{+}} \xi\left(2+\ln \frac{(\kappa a)^{2} z_{+}}{2\left(z_{+}+z_{-}\right)}\right)+\frac{1}{z_{+}}\left(\xi-\frac{1}{z_{+}}\right) \ln \left[\left(z_{+} \xi-1\right)^{2}+\widetilde{\mu}^{2}\right] \\
& +\frac{1}{z_{+}^{2}}\left(2 \ln 2-\widetilde{\mu}^{2}(\ln (\kappa a)+\mathcal{C})+\ln (\kappa a)+2 \mathcal{I}\right) .
\end{aligned}
$$

With $\widetilde{\mu}$ obtained from Eq. (18) (graphically shown in Fig. 2), Eq. (19) gives the grand potential for $\xi$ in the range close to $\xi_{c}$ (both below and above) and in all the range $\xi>\xi_{c}$ including highly charged cylinders. If one is interested only in the dependence of the grand potential on the salt concentration through the value of $\kappa a$, it can be checked that Eq. (19) reproduces the results from Eq. (13) of Ref. [11] which gives the grand potential per elementary charge $(g=\omega / \xi)$ dependency on $\kappa a$ for a 1:1 electrolyte only. However, in that work, all the dependency of $g$ on $\xi$ and on the electrolyte valencies was hidden in an arbitrary reference value (named $G_{\text {ref }}^{\text {coul }}$ in Ref. [11]) which was inaccessible analytically up until now. Our result, Eq. (19), provides more complete results with the complete $\xi$ dependence. It is also valid for other valencies $z_{-}: z_{+}$besides $1: 1$, provided two valency-dependent parameters are known

$$
\begin{aligned}
\mathcal{C} & =\Psi^{\prime}(0), \\
\mathcal{I} & =\int_{-i}^{0} \Psi(u) d u .
\end{aligned}
$$

In the cases 1:1, 1:2 and 2:1, these can be computed exactly,

$$
\begin{aligned}
& \mathcal{C}_{1: 1}=\gamma-3 \ln 2 \simeq-1.50223 \\
& \mathcal{C}_{1: 2}=\gamma-(3 \ln 3) / 2-(\ln 2) / 3 \simeq-1.30175 \\
& \mathcal{C}_{2: 1}=\gamma-(3 \ln 3) / 2-\ln 2 \simeq-1.76385
\end{aligned}
$$

and

$$
\begin{aligned}
& \mathcal{I}_{1: 1}=1-6 \ln \mathcal{A}-(\ln 2) / 3 \simeq-0.723576 \\
& \mathcal{I}_{1: 2}=1-6 \ln \mathcal{A}-\ln 2+(\ln 3) / 2 \simeq-0.636368 \\
& \mathcal{I}_{2: 1}=1-6 \ln \mathcal{A}-\ln 2+(5 \ln 3) / 16 \simeq-0.842358
\end{aligned}
$$

with $\gamma \simeq 0.577216$ the Euler Mascheroni constant and $\mathcal{A} \simeq 1.28243$ the Glaisher constant. The values of $\mathcal{C}$ were computed in [16] and the calculation of $\mathcal{I}$ is shown in appendix C. For other valencies, we computed numerically the 


\begin{tabular}{|c|c|c|c|}
\hline$z_{-}$ & $z_{+}$ & $\mathcal{C}$ & $\mathcal{I}$ \\
\hline 4 & 1 & -2.069 & -0.98620 \\
\hline 3 & 1 & -1.938 & -0.92408 \\
\hline 2 & 1 & -1.764 & -0.84236 \\
\hline 3 & 2 & -1.649 & -0.78950 \\
\hline 1 & 1 & -1.502 & -0.72358 \\
\hline 2 & 3 & -1.377 & -0.66861 \\
\hline 1 & 2 & -1.302 & -0.63637 \\
\hline 1 & 3 & -1.215 & -0.59988 \\
\hline 1 & 4 & -1.167 & -0.57969 \\
\hline
\end{tabular}

TABLE I. The constants $\mathcal{C}$ and $\mathcal{I}$ needed for the determination of the grand potential for different valencies.

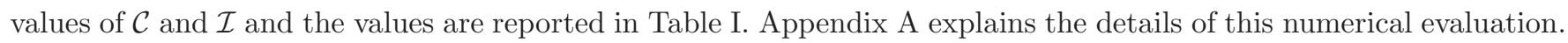
It should be kept in mind that up to a trivial rescaling, 1:1, 2:2, 3:3 etc. electrolytes are all equivalent within PB theory (only the ratio $z_{+} / z_{-}$does matter). Yet, upon increasing ionic valencies, correlation effects, discarded at the PB level, become more prevalent and may invalidate the mean-field assumption [23].

\section{RESULTS AND BENCHMARK OF THE ANALYTIC PREDICTIONS}

We benchmarked our analytic result against a direct numerical computation of the free energy. Details are given in Appendix A. Two methods have been used for the numerical calculation: either from Eq. (2) which requires to solve numerically a number of $\mathrm{PB}$ equations at fixed $\kappa a$ for a number of charges (starting from $\xi=0$ ), or alternatively from Eq. (B6) which only requires the solution of $\mathrm{PB}$ equation at the chosen values of $\xi$ and $\kappa a$. Checking that both methods yield identical results is important for assessing the validity of the calculations. Details of the numerical resolution of PB equation are given in appendix $\mathrm{A}$ Fig. 3 shows the grand potential per elementary charge, $g=\omega / \xi$, for the case $\kappa a=0.1$ and valencies 1:1 using several approximations: "analytic" stands for Eq. (19) with $\widetilde{\mu}$ obtained from Eq. (18), DH is Debye-Hückel prediction shown in Eq. (30), TW is the prediction from Ref. [13] recalled in appendix (D) Eq. (D5), and "large $\xi$ " is Eq. (19) with the approximation

$$
\widetilde{\mu} \simeq \frac{-\pi}{\ln (\kappa a)+\mathcal{C}+\left(z_{+} \xi-1\right)^{-1}},
$$

which is valid asymptotically for large $\xi \gg \xi_{c}$ [17]. The inset shows the relative error between the different predictions against the numerical calculation of the grand potential. Interestingly, there is a large overlap between the simple Debye-Hückel prediction

$$
g_{\mathrm{DH}}=\xi \frac{K_{0}(\kappa a)}{\kappa a K_{1}(\kappa a)},
$$

(where $K_{0}$ and $K_{1}$ are the modified Bessel functions of order 0 and 1), and the analytic prediction Eq. (19) with Eq. (18). For large $\xi \gg \xi_{c}$, Eq. (19), with either Eq. (18) or Eq. (29) for the determination of $\widetilde{\mu}$, provides extremely accurate results with a relative difference between the numerics and the analytic predictions that are below $0.2 \%$ $\left(\xi_{c} \simeq 0.737175\right.$ for $\left.\kappa a=0.1\right)$. This is 10 times more accurate than previous analytic predictions [1] for that range of values.

For $\xi<\xi_{c}$, the solution for $\widetilde{\mu}$ involves the region $\zeta>1$ of $h(\zeta)$ (see Figure 21). In this region $|\widehat{\mu}|$ increases faster, therefore the range of validity of the approximation $|\widetilde{\mu}| \ll 1$ is smaller. Nevertheless, the analytic prediction of Eq. (19) with Eq. (18) remains accurate for values of $\xi$ smaller than $\xi_{c}$ provided $\xi_{c}-\xi \ll 1$. In practice, Eq. (19) can be applied down to values of $\xi=1 /\left(2 z_{+}\right)$(half the Manning parameter $1 / z_{+}$) with an error that starts to become larger than $1 \%$ below that threshold. Then, for smaller values of $\xi$ the most accurate analytic expression is provided by the Debye-Hückel prediction (30). It turns out that the analytic expression from Ref. [13] recalled in Eqs. (D5)-(D7) is less accurate than the $\mathrm{DH}$ prediction. This is probably traced back to the fact that $\mathrm{DH}$ prediction gives the correct asymptotics for $\xi \rightarrow 0$ of the nonlinear PB problem regardless of the value of $\kappa a$ (it is not limited to $\kappa a \ll 1$ ). In summary, our main result Eq. (19) with Eq. (18) for moderate to highly charged polyion, combined with DH prediction (30) for smaller values of $\xi$ provide excellent accurate analytic predictions for the grand potential. 


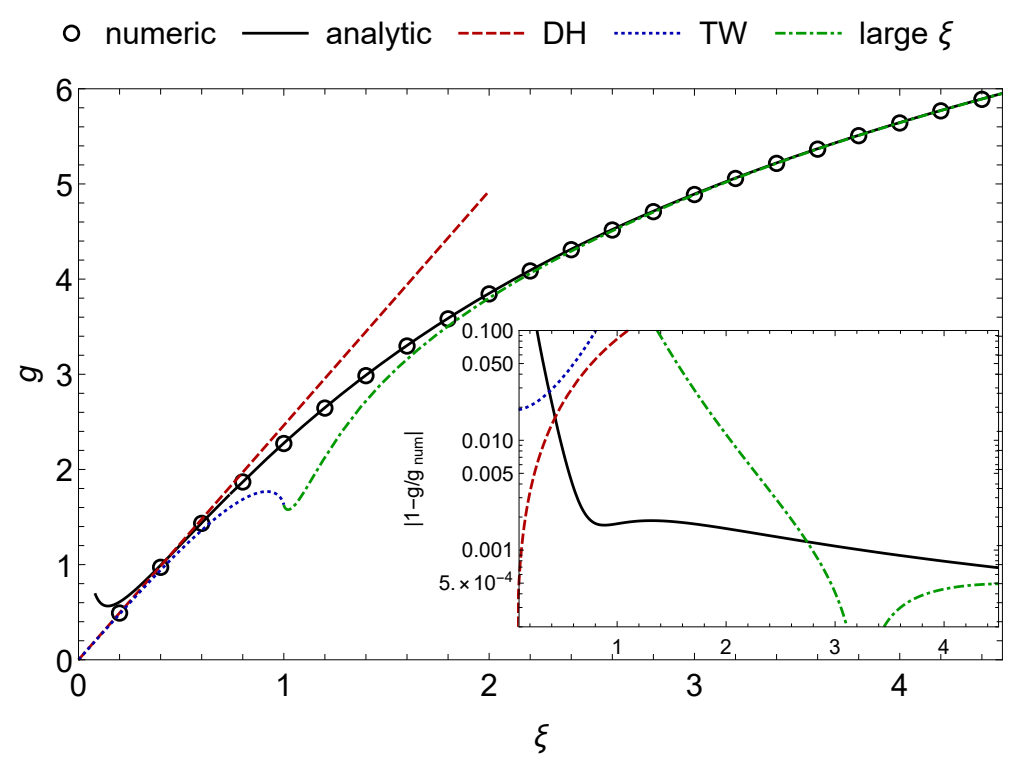

FIG. 3. Comparison of the different predictions for the grand potential $g$ for $\kappa a=0.1$ and $1: 1$ electrolyte as a function of $\xi$ : "analytic" is Eq. (19) with Eq. (18), "DH" is Debye-Hückel prediction Eq. (30), "TW" is Tracy and Widom prediction Eq. (D5) and "large $\xi$ " is Eq. (19) but with $\widetilde{\mu}$ approximated as shown in Eq. (18). Inset: Relative error between the analytic predictions and the numeric result of the grand potential.
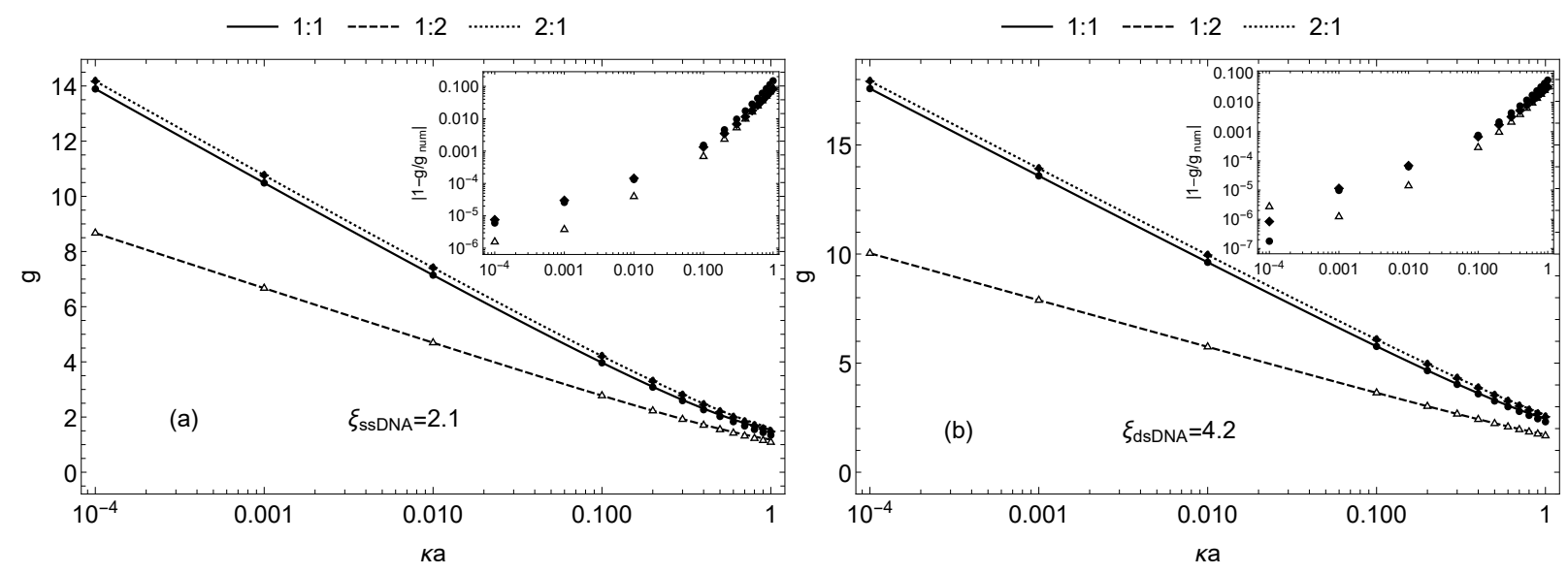

FIG. 4. Grand potential as a function of $\kappa a$ for (a) ssDNA and (b) dsDNA. The lines are the analytic predictions and the symbols the numerical evaluation. Inset: relative error between the analytic prediction and the numerical evaluation. The grand potential is computed per elementary charge $g=\omega / \xi$.

To test the accuracy of our prediction when $\kappa a$ varies, we consider two experimentally relevant cases corresponding to single stranded DNA $\xi=\xi_{\text {ssDNA }}=2.1$ and double stranded DNA $\xi=\xi_{\text {dsDNA }}=4.2$. Fig. 团 shows the grand potential as a function of $\kappa a$ (obtained varying salt concentration) with a comparison to the numerical evaluation. The insets of the figures show the relative error between the two. The worst case is for 1:1 electrolyte and ssDNA where the error reaches values beyond $1 \%$ but only for $\kappa a \geq 0.4$. Even when $\kappa a=1$, where the analytic treatment is not supposed to be accurate, we obtained a fair approximation for the grand potential with relative error below $3.5 \%$ for a 1:2 and 2:1 electrolytes for dsDNA.

For highly charged polyions, the Debye-Hückel theory has often been applied by correcting the bare charge with the effective one which encodes the large distance features of the electric potential [10, 16, 24, 25]. Fig. 5 compares the analytic result with this prescription, showing that, for the free energy and grand potential calculations, the effective charge concept as previously formulated fails. In particular, since the effective charge saturates for highly charged polyions, it would predict that the grand potential per elementary charge will saturate, when in reality this is not the 


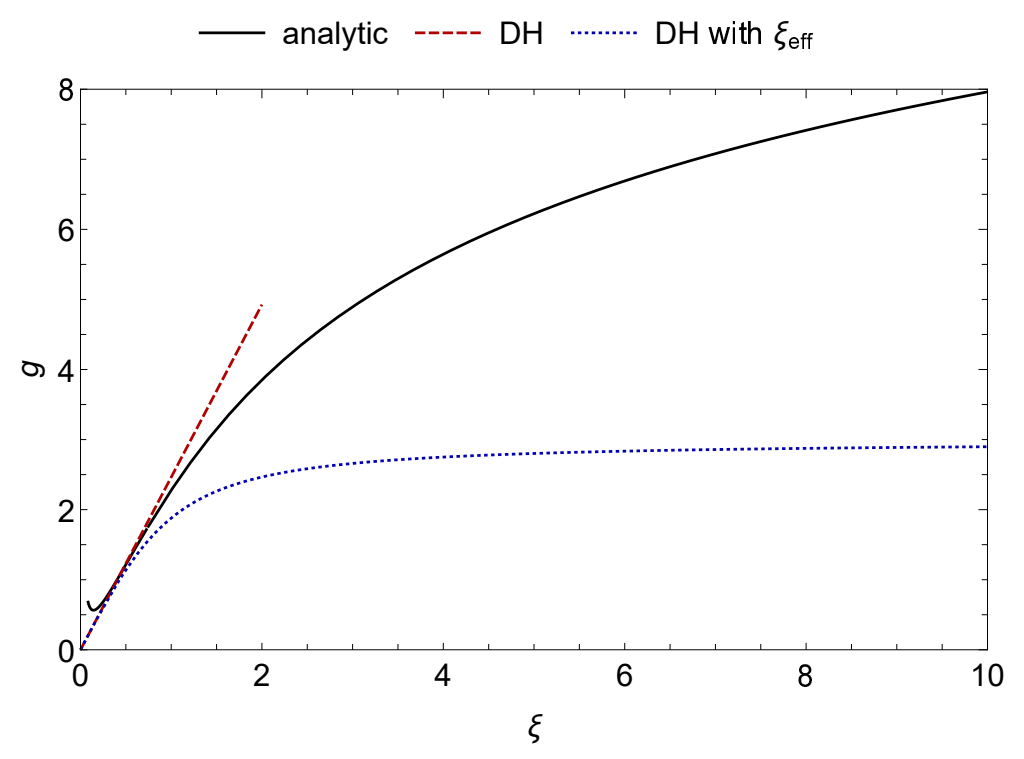

FIG. 5. Failure of the prediction for the grand potential $g$ using the Debye-Hückel theory combined with effective charge corrections $\xi_{\text {eff. }}$ The data is shown for $\kappa a=10^{-1}$ and 1:1 electrolyte and compared to the analytic results Eq. (19).

case. Our analytic results (19) predict that for highly polyions, the grand potential behaves as

$$
g=\frac{1}{z_{+}}\left(2 \ln \xi-2-\ln \frac{(\kappa a)^{2}}{2\left(z_{+}+z_{-}\right) z_{+}}\right)+O\left(\xi^{-1} \ln \xi\right), \quad \xi \rightarrow \infty .
$$

The failure of the effective charge prescription for the computation of the grand potential is probably due to the strong free energy contribution of the condensed ions i.e. of short scale features ignored by the far-field behaviour subsumed in the effective charge.

We now discuss the dependency of the grand potential on the valencies $z_{-}: z_{+}$of the electrolyte. Using our prediction Eq. (19) and the data from Table \obtained in appendix $\mathrm{A}$ we plot $g$ as a function of $\xi$ when the valency is changed (Fig. 6). The salt concentration is fixed at $\kappa a=10^{-1}$. Note how the different curves can be regrouped by common counterion valency $z_{+}$. At fixed counterion valency $\left(z_{+}\right)$, when the coion valency $z_{-}$is increased, the grand potential increases moderately. On the other hand, an increase on the counterion valency $z_{+}$reflects in a large decrease on the grand potential. This is also apparent on the analytic expression (19), where it can be appreciated that the dependency on $z_{-}$is logarithmic while there are terms proportional to $z_{+}$and $z_{+}^{2}$ responsible of a stronger dependency on $z_{+}$than on $z_{-}$. Fig. 7 confirms this trend, showing now $g$ for dsDNA as a function of $\kappa a$ for different valencies. From Eqs. (19) and (31), one can notice that at fixed large $\xi$, the leading behavior dependence on $\kappa a$ is $g \sim-\left(2 / z_{+}\right) \ln (\kappa a)+O(1)$ for $\kappa a \ll 1$. This linear dependence of $g$ on $\ln (\kappa a)$ at leading order is verified on Fig. 7 where the slope of the curves in $\log$-scale is indeed $-2 / z_{+}$. The coion valency $z_{-}$only affects the subleading order terms.

\section{SUMMARY AND CONCLUSION}

Coulomb interactions are key to rationalizing the thermodynamics of nucleic acid processes [1], or other properties of biopolymers such as their persistence length [26]. The non-linear Poisson-Boltzmann (PB) theory adopted here is a mean-field framework that provides a useful description, not only in the present context but more generally for studying soft matter in aqueous solutions, where Coulombic effects are paramount [1 3 ]. We computed the exact analytic low-salt asymptotic expansion of the grand potential/free energy of a long cylindrical polyion (Eqs. (19) and (18)). The biopolymer is thus modeled here are as a uniformly charged straight cylinder, and we addressed the case of a binary electrolyte, with arbitrary co/counter ion valencies $z_{-}$and $z_{+}$. Analytical progress was possible taking advantage of the contact potential (3) derived in previous works [17] and writing expressions valid for all values of polyion charge $\xi$. This required to introduce an auxiliary quantity, $\widetilde{\mu}$, appropriately allowed to take complex values, either pure imaginary or real, as sketched in Fig. 1. This results in a significantly extended range of validity of the contact surface potential and the quantities derived from it, such as the preferential interaction coefficient computed in Ref. [17], and the grand potential computed here. With this, one can obtain reliable results for moderate to 


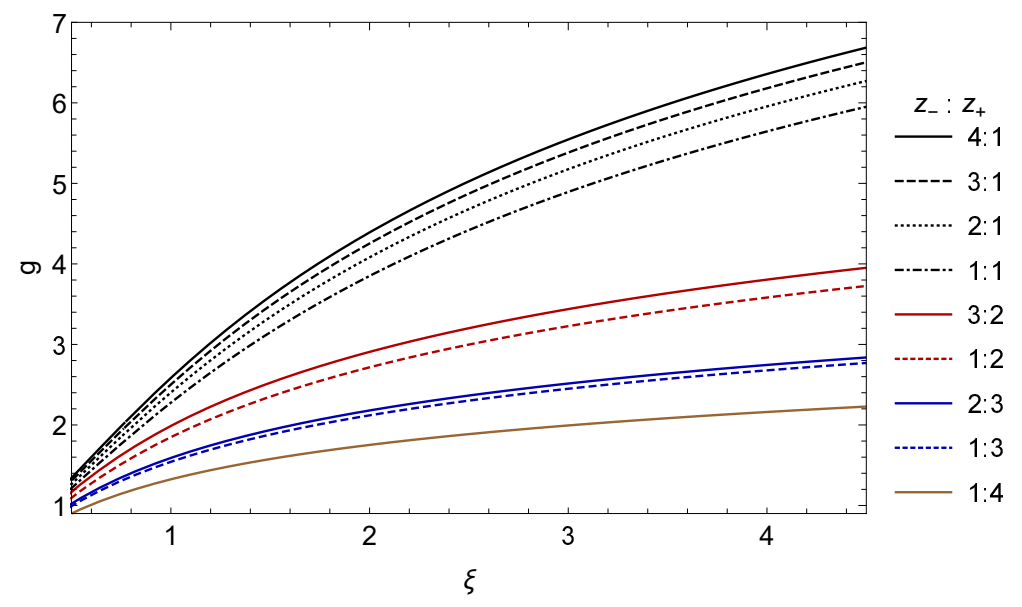

FIG. 6. Grand potential per elementary charge $g$ as a function of $\xi$ for different valencies $z_{-}: z_{+}$at $\kappa a=10^{-1}$.

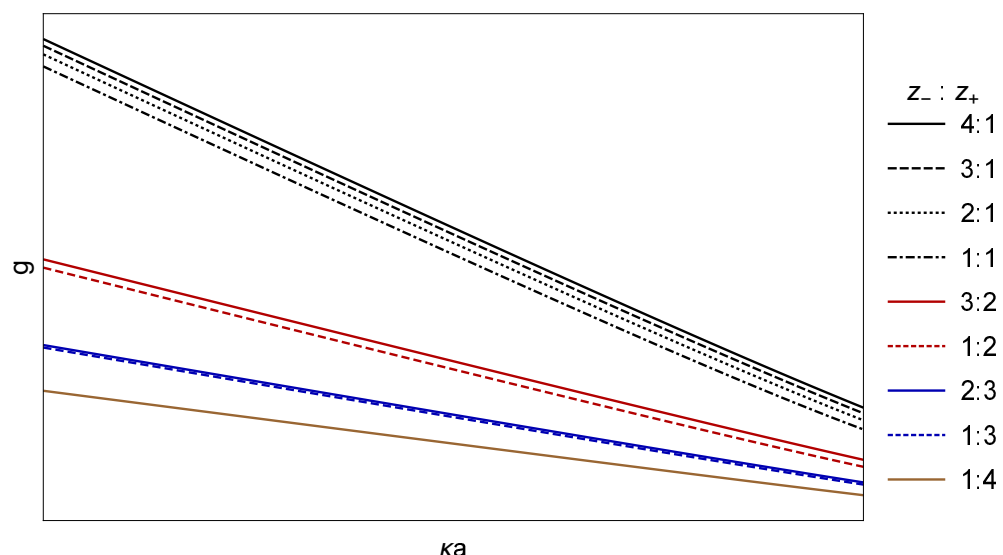

FIG. 7. Grand potential per elementary charge $g$ as a function of $\kappa a$ for different valencies $z_{-}: z_{+}$at $\xi=\xi_{\text {dsDNA }}=4.2$.

highly charged polyions, having linear charge $\xi$ larger than half the Manning parameter $\left(\xi>1 /\left(2 z_{+}\right)\right.$, therefore $1 / 2$ for monovalent counterions). The regime of smaller charges is somewhat less interesting: for smaller values of $\xi$, one enters the realm of the simple linear Debye-Hückel theory, which provides accurate results. This opens the opportunity to present the analytic results for the cylindrical PB equation 13, 15 17] in a unified framework, that no longer requires to consider different formulas for $\xi<\xi_{c}$ and $\xi \geq \xi_{c}$, as done in previous publications. In practice, our low salt approach turns reliable for $\kappa a<1$. We finally emphasize that our work sheds some light into the analytic properties of the grand potential. Within the present PB formalism, it turns out to be a holomorphic function of $\widetilde{\mu}$ and $\xi$, with no singularity even at $\xi=\xi_{c}$, unlike what the widespread terminology pertaining to Manning "condensation transition" may lead to believe.

\section{ACKNOWLEDGMENTS}

This work is partially funded by ECOS-Nord action C18P01. G.T. acknowledges support from Fondo de Investigaciones, Facultad de Ciencias, Universidad de los Andes, Research Program 2018-2019 "Modelos de baja dimensionalidad de sistemas cargados". 


\section{Appendix A: Numerical evaluation of the grand potential}

In this appendix we give a few details for algorithm to compute the grand potential/free energy. The numerical resolution of PB equation was done with MATHEMATICA based on the code presented in Appendix A of Ref. [10]. Essentially, PB equation is solved on a cylindrical Wigner-Seitz cell of large radius $R=22 \kappa^{-1}$ with boundary conditions at the edge of the cell $\phi^{\prime}(R)=0$ (by electroneutrality) and a test value for the potential at the edge $\phi(R)=\phi_{\text {edge }}$. The resolution of the differential equation is done with MATHEMATICA NDSolve built-in algorithm with options MaxSteps $\rightarrow 500$ and WorkingPrecision $\rightarrow 33$. We are interested in the $R \rightarrow \infty$ limit (infinite dilution) and $R$ should be chosen accordingly, large enough to provide an acceptable solution. It is then necessary to check that the results obtained do not depend on $R$, within the targeted accuracy.

If the algorithm converges successfully, the corresponding linear charge can be obtained as $\xi=a \phi^{\prime}(a) / 2$. In the

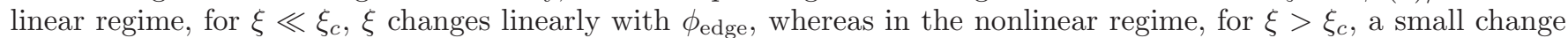
on $\phi_{\text {edge }}$ produces exponentially large changes on $\xi$. Due to the potential saturation effect [27], if $\phi_{\text {edge is too large }}$ (beyond its saturation value $\phi_{\text {sat }}$ ), the algorithm will not converge. By trial and error, the saturation value of $\phi_{\text {edge }}$

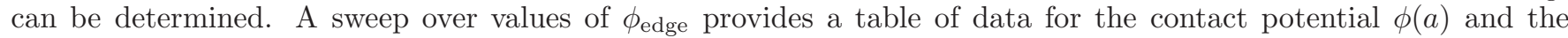
corresponding linear charge value $\xi$. This sweep should be done with equal spacing on a log scale of values of $\phi_{\text {edge }}$ starting at $\phi_{\text {sat }}$ to account for the saturation effect to obtain linearly evenly spaced for values of $\xi$. In practice, we used $\phi_{\text {edge }}=\phi_{\text {sat }}\left(1-(97 / 100)^{k}\right)$ with $k$ ranging from 0 to 400 by step increments of 5 . This produces a table of 80 values of the contact potential and its corresponding linear charge. This table is interpolated to produce numerically the function $\xi \mapsto \phi_{0}$. The interpolation was made with MATHEMATICA Interpolation function with default options (degree 3 polynomial interpolation between successive data points). This function is then integrated numerically with MAтнемAтісA Integrate to obtain the grand potential (Eq. (2)). The above procedure is followed for a given value of $\kappa a$ and gives the grand potential for any value of $\xi$. If $\kappa a$ is changed the procedure should be run again since the saturation value $\phi_{\text {sat }}$ changes. Besides, an important test for the correctness of the calculation is to check that the grand potential values are recovered by a direct calculation, without any integral over $\xi$, making use of relation (B6) below. Our results satisfied this test.

The numerical evaluation of the constants $\mathcal{C}\left(\mathrm{Eq} \cdot(20)\right.$ ) and $\mathcal{I}$ (Eq. (21)) for different valencies $z_{-}: z_{+}$was done as follows. Using Eq. (13) into Eq. (3), shows that the contact potential $\phi_{0}$ and the parameter $\widetilde{\mu}$ satisfy

$$
e^{-z_{+} \phi_{0}} \frac{(\kappa a)^{2} z_{+}}{2\left(z_{+}+z_{-}\right)}-\left(z_{+} \xi-1\right)^{2}=\widetilde{\mu}^{2}
$$

Therefore, the left-hand side (LHS) of Eq. (A1) vanishes when $\xi=\xi_{c}(\widetilde{\mu}=0)$. Along with the numerical computation of the grand potential explained above, a data table of the LHS of Eq. (A1) as a function of $\xi$ can be build then interpolated. The zero of this interpolated function the closest to $1 / z_{+}$is then found using MATHEMATICA FindRoot algorithm to obtain $\xi_{c}$. With $\xi_{c}$ determined numerically, the constant $\mathcal{C}$ is obtained from Eq. (6). The constant $\mathcal{I}$ is obtained from Eq. (19) evaluated at $\xi=\xi_{c}$ (corresponding to $\widetilde{\mu}=0$ ). A strong test of this algorithm is that it should give the same values of $\mathcal{C}$ and $\mathcal{I}$ independently of the chosen value of $\kappa a$ provided it is small enough. We tested this numerical procedure using values of $\kappa a=10^{-6}, 10^{-5}$ and $10^{-4}$, confirmed the stability of the numerical values of $\mathcal{C}$ and $\mathcal{I}$ and reproduced the analytically known values for the solvable cases of valency 1:1, 1:2 and 2:1 with a relative accuracy of $10^{-5}$ for $\mathcal{C}$ and $10^{-6}$ for $\mathcal{I}$. Table $\square$ provides the values of $\mathcal{C}$ and $\mathcal{I}$ for other valencies of experimental interest, bearing in mind the limitations of PB framework for larger valencies [23].

\section{Appendix B: Charging process to obtain the free energy}

Several charging processes can be put forward to compute the free energy by studying its variations with respect to different parameters. A review of such process can be found in [6, 18 20]. Consider the cell model and PB theory for a polyion. In [19] it is shown that the variations of the free energy $F$ are given by

$$
\delta(\beta F)=\frac{1}{8 \pi l_{B}} \oint_{\Sigma}(\phi \nabla(\delta \phi)-\delta \phi \nabla \phi) \cdot d S+\beta U \frac{\delta l_{B}}{l_{B}}+\int_{\mathcal{P}} \phi \delta\left(\frac{\sigma}{e}\right) d S+\sum_{s= \pm} \ln \left(n_{s}^{0} \Lambda_{s}^{3}\right) \delta N_{s}
$$

where $\mathcal{P}$ is the surface of the polyion, $U$ the internal energy, $N_{ \pm}$the number of positive and negative ions, $\sigma$ the surface charge density of the polyion, and $\Sigma$ is the surface of the Wigner-Seitz cell of arbitrary shape that encloses the system; in the present situation of infinite dilution, $\Sigma$ is "sent to infinity" and the corresponding integral is absent from the equation, as a consequence of screening; the spatial integrals considered consequently run over all space. Besides, the bulk electrolyte plays the role of a reservoir, with given chemical potentials $\mu_{ \pm}$for cations and anions. It 
is thus appropriate to work in the grand canonical ensemble due to the chemical equilibrium with the salt reservoir. Therefore, we consider the grand potential $\Omega=F-\mu_{+} N_{+}-\mu_{-} N_{-}$and its excess value with respect to that of the reservoir $\Omega_{0}=\int\left(n_{0}^{+}+n_{0}^{-}\right) d V$. At fixed $l_{B}$, its variations are

$$
\delta\left(\beta\left(\Omega-\Omega_{0}\right)\right)=\int_{\mathcal{P}} \phi \delta\left(\frac{\sigma}{e}\right) d S-\int\left(n^{+}(\mathbf{r})-n_{0}^{+}\right) \delta \mu_{+} d V+\int\left(n^{-}(\mathbf{r})-n_{0}^{-}\right) \delta \mu_{-} d V,
$$

where $n^{ \pm}(\mathbf{r})$ is the ionic density profiles around the polyion. The second and third term show that the variations with respect to the chemical potentials are the excess ionic charge around the polyion, which is essentially the preferential interaction coefficient [17, 28]. Therefore, the grand potential can be obtained by integrating the preferential interaction coefficient with respect to the chemical potential $\mu_{ \pm}=\ln \left(n_{0}^{ \pm} \Lambda_{ \pm}^{3}\right)$ or equivalently with respect to $\ln (\kappa a)$. This strategy was used in [11] to obtain analytic predictions for the grand potential. However, it has the disadvantage that it requires the determination of an arbitrary reference value of the grand potential at a given salt density (0.15 $\mathrm{M}$ was used in [11]). This reference value is different for each value of $\xi$ even if $\kappa a$ is kept fixed. In this work, we followed another route by considering variations of the surface charge $\sigma$ of the polyion. This is equivalent to varying $\xi$ since $\sigma=-\xi e /\left(2 \pi a l_{B}\right)$. For a cylinder of length $L$, at fixed chemical potentials, Eq. (B2) becomes

$$
\delta\left(\beta\left(\Omega-\Omega_{0}\right)\right)=-\frac{L}{l_{B}} \phi_{0} \delta \xi .
$$

Let us define the dimensionless excess grand potential per unit length

$$
\omega=\beta\left(\Omega-\Omega_{0}\right) l_{B} / L
$$

and the dimensionless excess grand potential per elementary charge

$$
g=\beta\left(\Omega-\Omega_{0}\right) / N=\omega / \xi,
$$

with $N=L / b$ the number of elementary charges of the polyion. Since at $\xi=0$ the grand potential is $\Omega_{0}$, we obtain (2) from (B3).

For putting to the test the reliability of our numerical solution, we have computed the free energy/grand potential through an alternative route, that does not require any $\xi$-integration. Once $\xi$ and $\kappa a$ have been chosen and $\mathrm{PB}$ equation solved, we have [19]

$$
\beta \omega=-\frac{1}{2} \xi \phi_{0}+\frac{\kappa^{2}}{8 \pi\left(z_{+}+z_{-}\right)}\left(\int \phi\left(e^{z_{-} \phi}-e^{-z_{+} \phi}\right) d^{2} V-2 \int\left[\frac{1}{z_{-}}\left(e^{z_{-} \phi}-1\right)+\frac{1}{z_{+}}\left(e^{-z_{+} \phi}-1\right)\right] d^{2} V\right),
$$

where the second integral on the right hand side is a rewriting of $\int\left(n^{+}+n^{-}-n_{0}^{+}-n_{0}^{-}\right) d^{2} V$. The two integrals in (B6) run over the 2D plane perpendicular to the cylinder axis, outside the charged cylinder $(r>a)$.

\section{Appendix C: Explicit solutions for 1:1, 1:2 and 2:1 valencies}

In the short distance asymptotics of the electrostatic potential from which the contact potential is deduced (Eq. (3)), $\widetilde{\mu}$ and $\Psi(\widetilde{\mu})$ are the two constants of integration of the differential equation (1). However, to satisfy the boundary condition $r \phi^{\prime}(r) \rightarrow 0$ when $r \rightarrow \infty$, the so-called connection problem between the short and large scale behavior of the solution has to be solved to find the relationship between the two integration constants. This problem was worked out in the integrable cases of valencies $1: 1,1: 2,2: 1$, where the solution to $\mathrm{PB}$ equation can be written down in terms of Fredholm determinants [12, 14]. We recall here the main results. For those valencies, the constant $B$ appearing in Eq. (41) is given by [29]

$$
\begin{aligned}
& B_{1: 1}=2^{6 A} \gamma\left(\frac{1+A}{2}\right)^{2} \\
& B_{1: 2}=3^{3 A} 2^{2 A} \gamma\left(\frac{1+2 A}{3}\right) \gamma\left(\frac{2+A}{3}\right) \\
& B_{2: 1}=3^{3 A} 2^{2 A} \gamma\left(\frac{2(1+A)}{3}\right) \gamma\left(\frac{1+A}{3}\right)
\end{aligned}
$$


where $\gamma(z)=\Gamma(z) / \Gamma(1-z)$ with $\Gamma$ the Euler gamma function. With $A$ related to $\tilde{\mu}$ by $i \tilde{\mu}=1+z_{+} A$, replacing this in Eq. (4) gives the function $\Psi$

$$
\begin{aligned}
& \Psi_{1: 1}(\widetilde{\mu})=-3 \widetilde{\mu} \ln 2+i \ln \left(\frac{i \widetilde{\mu}}{2} \gamma\left(\frac{i \widetilde{\mu}}{2}\right)\right) \\
& \Psi_{1: 2}(\widetilde{\mu})=-\frac{\widetilde{\mu}}{2}(3 \ln 3+2 \ln 2)+i \ln \left(\frac{i \widetilde{\mu}}{3} \gamma\left(\frac{i \widetilde{\mu}}{3}\right)\right)+i \ln \gamma\left(\frac{i \widetilde{\mu}+3}{6}\right) \\
& \Psi_{2: 1}(\widetilde{\mu})=-\frac{\widetilde{\mu}}{2}(3 \ln 3+2 \ln 2)+\frac{i}{2} \ln \left(\frac{i \widetilde{\mu}}{3} \gamma\left(\frac{i \widetilde{\mu}}{3}\right)\right)+\frac{i}{2} \ln \left(\frac{2 i \widetilde{\mu}}{3} \gamma\left(\frac{2 i \widetilde{\mu}}{3}\right)\right) .
\end{aligned}
$$

With this, the constant $\mathcal{I}=\int_{-i}^{0} \Psi(\widetilde{\mu}) d \widetilde{\mu}$ used in Eq. (19) can be computed explicitly, leading to Eqs. (25)-(27).

\section{Appendix D: Results for $\xi$ below the critical value $\xi_{c}$}

Section [II main result Eq. (19) breaks down if $\xi \ll \xi_{c}$. We develop here an approximation appropriate for that range. When $\xi<\xi_{c}, \widetilde{\mu} \in[-i, 0]$, therefore it is useful to introduce $\nu \in[-1,0]$ defined by $\widetilde{\mu}=i \nu$. Eq. (50) becomes

$$
z_{+} \xi-1=\nu \operatorname{coth}(\nu \ln (\kappa a)+\varphi(\nu))
$$

with $\varphi(\nu)=-i \Psi(\widetilde{\mu}) \in \mathbb{R}$. For $\kappa a \ll 1$, the argument of the hyperbolic cotangent in (D1) is large and positive. Therefore,

$$
z_{+} \xi-1=\nu\left(1+2 e^{-2 \nu \ln (\kappa a)+\varphi(\nu)}+o\left((\kappa a)^{-2 \nu}\right)\right.
$$

which yields

$$
\nu=\left(z_{+} \xi-1\right)\left(1-2(\kappa a)^{2\left(1-z_{+} \xi\right)} e^{-2 \varphi\left(z_{+} \xi-1\right)}+o\left((\kappa a)^{2\left(1-z_{+} \xi\right)}\right) .\right.
$$

Replacing this in Eq. (14) and using Eq. (4) we find

$$
\omega=-\xi^{2} \ln (\kappa a)-\frac{1}{z_{+}} \int_{-1}^{-1+z_{+} \xi} \ln B d \nu,
$$

where $B$ should be seen as a function of $\nu=-\left(1+z_{+} A\right)$. This is the same result as if one starts with the approximation $\phi_{0}=-2 A \ln (\kappa a)+\ln B$, with $A$ approximated as $A=-\xi$. Further progress can be only made in the cases of valencies 1:1, 1:2 and 2:1, where $B$ is explicitly known [12, 14] (see Eqs. (C1)-C3) from appendix C), recovering previous results from Tracy and Widom Ref. [13],

$$
\begin{aligned}
\omega_{1: 1}= & \xi^{2}(-\ln (\kappa a)+3 \ln 2)+4\left[\psi^{(-2)}\left(\frac{1-\xi}{2}\right)+\psi^{(-2)}\left(\frac{1+\xi}{2}\right)-2 \psi^{(-2)}\left(\frac{1}{2}\right)\right] \\
\omega_{1: 2}= & \xi^{2}\left(-\ln (\kappa a)+\frac{3}{2} \ln 3+\ln 2\right)+3\left[\frac{1}{2}\left(\psi^{(-2)}\left(\frac{2(1+\xi)}{3}\right)+\psi^{(-2)}\left(\frac{1-2 \xi}{3}\right)\right)+\psi^{(-2)}\left(\frac{1+\xi}{3}\right)+\psi^{(-2)}\left(\frac{2-\xi}{3}\right)\right. \\
& \left.-\frac{3}{2}\left(\psi^{(-2)}\left(\frac{2}{3}\right)+\psi^{(-2)}\left(\frac{1}{3}\right)\right)\right] \\
\omega_{2: 1}= & \xi^{2}\left(-\ln (\kappa a)+\frac{3}{2} \ln 3+\ln 2\right)+3\left[\frac{1}{2}\left(\psi^{(-2)}\left(\frac{2(1-\xi)}{3}\right)+\psi^{(-2)}\left(\frac{1+2 \xi}{3}\right)\right)+\psi^{(-2)}\left(\frac{1-\xi}{3}\right)+\psi^{(-2)}\left(\frac{2+\xi}{3}\right)\right. \\
& \left.-\frac{3}{2}\left(\psi^{(-2)}\left(\frac{2}{3}\right)+\psi^{(-2)}\left(\frac{1}{3}\right)\right)\right]
\end{aligned}
$$

where $\psi^{(-2)}(x)=\int_{0}^{x} \ln \Gamma(u) d u[30,31]$.

[1] Y. Levin, Rep. Prog. Phys. 65, 1577 (2002).

[2] D. Andelman, in Soft Condensed Matter Physics in Molecular and Cell Biology, edited by W. Poon and D. Andelman (Addison Wesley, 2006) Chap. 6.

[3] R. Messina, J. Phys.: Condens. Matter 21, 113102 (2009). 
[4] E. J. W. Verwey and J. T. G. Overbeek, Theory of the stability of lyophobic colloids (Elsevier, 1948).

[5] D. Y. Chan and D. Mitchell, Journal of Colloid and Interface Science 95, 193 (1983)

[6] D. Stigter and K. A. Dill, The Journal of Physical Chemistry 93, 6737 (1989)

[7] I. A. Shkel, J. D. Ballin, and M. T. Record, Biochemistry 45, 8411 (2006)

[8] M. L. Bret and B. H. Zimm, Biopolymers 23, 287 (1984).

[9] P. Mills, C. F. Anderson, and M. T. Record, The Journal of Physical Chemistry 89, 3984 (1985).

[10] E. Trizac, L. Bocquet, M. Aubouy, and H. H. von Grünberg, Langmuir 19, 4027 (2003)

[11] I. A. Shkel, The Journal of Physical Chemistry B 114, 10793 (2010).

[12] B. M. McCoy, C. A. Tracy, and T. T. Wu, J. Math. Phys. 18, 1058 (1977).

[13] C. A. Tracy and H. Widom, Physica A: Statistical Mechanics and its Applications 244, 402 (1997).

[14] C. A. Tracy and H. Widom, Commun. Math. Phys. 190, 697 (1998).

[15] E. Trizac and G. Téllez, Phys. Rev. Lett. 96, 038302 (2006).

[16] G. Téllez and E. Trizac, Journal of Statistical Mechanics: Theory and Experiment 2006, P06018 (2006).

[17] E. Trizac and G. Téllez, Macromolecules 40, 1305 (2007)

[18] R. A. Marcus, The Journal of Chemical Physics 23, 1057 (1955).

[19] E. Trizac and J.-P. Hansen, Journal of Physics: Condensed Matter 8, 9191 (1996)

[20] E. Trizac and J.-P. Hansen, Phys. Rev. E 56, 3137 (1997)

[21] R. M. Fuoss, A. Katchalsky, and S. Lifson, Proc Natl Acad Sci U S A 37, 579 (1951).

[22] T. Alfrey Jr., P. W. Berg, and H. Morawetz, Journal of Polymer Science 7, 543 (1951).

[23] R. Netz, Eur. Phys. J. E 5, 557 (2001).

[24] S. Alexander, P. M. Chaikin, P. Grant, G. J. Morales, P. Pincus, and D. Hone, The Journal of Chemical Physics 80, 5776 (1984).

[25] M. Aubouy, E. Trizac, and L. Bocquet, Journal of Physics A: Mathematical and General 36, 5835 (2003).

[26] E. Trizac and T. Shen, EPL (Europhysics Letters) 116, 18007 (2016).

[27] G. Téllez and E. Trizac, Phys. Rev. E 68, 061401 (2003).

[28] I. A. Shkel, O. V. Tsodikov, and J. Record, M. T., Proc. Natl. Acad. Sci. U.S.A. 99, 2597 (2002).

[29] In Ref. [17] the cases 1:2 and 2:1 were inverted in Eq. (D2).

[30] V. S. Adamchik, Journal of Computational and Applied Mathematics 100, 191 (1998)

[31] J. Choi and H. Srivastava, Journal of Mathematical Analysis and Applications 303, 436 (2005). 